Table. Proportion of TAB-positive GCA stratified by the main covariates.

\begin{tabular}{lcccc}
\hline Study and pt. characteristics & & $\begin{array}{c}\text { No. of studies } \\
\text { (pt.) }\end{array}$ & $\begin{array}{c}\text { Positive TAB } \\
(\%) \\
(95 \% \mathrm{Cl})\end{array}$ & $\mathrm{I}^{2}(\%)$ \\
\hline Mean age at diagnosis & $<72.7$ yrs & $10(961)$ & $81.2(71.1-88.4)$ & 91 \\
\hline$\geq 72.7$ yrs & $11(1067)$ & $74.8(65.1-82.6)$ & 91 & \\
Females & $<71 \%$ of pt. & $12(1156)$ & $78.3(67.0-86.5)$ & 94 \\
& $\geq 71 \%$ of pt. & $11(1319)$ & $81.6(73.9-87.4)$ & 88 \\
Polymyalgia rheumatica & $<42 \%$ of pt. & $7(629)$ & $70.6(59.4-79.9)$ & 87 \\
& $\geq 42 \%$ of pt. & $7(1024)$ & $79.2(66.2-88.0)$ & 94 \\
Ophthalmological signs & $<21 \%$ of pt. & $6(893)$ & $81.8(66.2-91.2)$ & 95 \\
& $\geq 21 \%$ of pt. & $7(606)$ & $72.2(60.4-81.6)$ & 87 \\
Large-vessel & $<25 \%$ of pt. & $2(373)$ & $63.0(50.6-73.8)$ & 78 \\
involvement & $\geq 25 \%$ of pt. & $2(203)$ & $61.1(54.2-67.5)$ & 0 \\
Study purpose & Diagnostic & $3(153)$ & $88.8(68.5-96.7)$ & 75 \\
Study type & Other & $29(2939)$ & $76.0(70.4-80.9)$ & 90 \\
& Retrospective & $24(2688)$ & $75.5(69.3-80.8)$ & 91 \\
Year of publication & Prospective & $8(404)$ & $83.1(70.6-90.9)$ & 86 \\
& $<2012$ & $16(1806)$ & $84.4(77.1-89.6)$ & 92 \\
& $\geq 2012$ & $16(1286)$ & $68.4(63.4-72.9)$ & 69 \\
\hline
\end{tabular}

Pt : patients

Disclosure of Interests: Emma Rubenstein: None declared, Carla Maldini: None declared, Solange GONZALEZ-CHIAPPE: None declared, Sylvie Chevret: None declared, Alfred Mahr Consultant for: Chugai Pharma France, Speakers bureau: Roche SAS Chugai Pharma France DOI: 10.1136/annrheumdis-2019-eular.4204

\section{SAT0243 EFFECTS OF TOFACITINIB SUPPRESSED PULMONARY VASCULAR REMODELING OF ALLERGIC VASCULITIS IN A MURINE MODEL}

Nobuhito Sasaki, Kohei Yamauchi. Iwate Medical University School of Medicine, Morioka, Japan, Division of Pulmonary medicine, Allergy and Rheumatology, Department of Internal Medicine, Morioka, Japan

Background: We reported allergic granulomatous vasculitis with eosinophil infiltration in an asthma model of $\mathrm{C} 57 \mathrm{BL} / 6$ sensitized with ovalbumin (OVA). TGF-btea and IL-6 are thought to play an important role in fibroblasts proliferation and is critical to vascular remodeling in vasculitis. Tofacitinib inhibits vascular endothelial cells proliferation and canalization. Objectives: To elucidate the role of tofacitinib in vascular remodeling of allergic granulomatous vasculitis, we examined the effects of tofacitinib on the vasculitis of the murine model.

Methods: C57BL/6 mice (6-8 weeks) were sensitized with ovalbumin (OVA) and alum. The positive controls $(n=9)$ were exposed to aerosolized OVA daily for 7 days. The other group of mice (tofacitinib treated mice $(n=9))$ were administered with tofacitinib $(100 \mathrm{mg} / \mathrm{kg}$ intraperitoneal administration) in parallel with daily exposure to aerosolized OVA for 7 days. On 7th day, bronchoalveolar lavage (BALF) was performed and the lungs were excised for pathological analysis. Cytokines in BALF were measured.

Results: The total cell number and the number of. Eosinophils in BALF on the 7th day were decreased significantly in the tofacitinib-treated mice compared with those of the control-positive mice. The blood eosinophil counts in the positive control increased after OVA inhalation. The blood eosinophil counts in the tofacitinib treated mice were lower on the than those in the positive control.
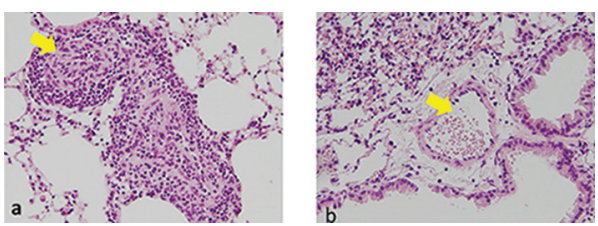

Figure 1. a. Positive control: Totally occluded pulmonary artery by intraluminal myofibroblasts in the OVA-sensitized mice with exposure to OVA in 7th day. (HE staining) b. Tofacitinib: Intraluminal myofibroblast accumulation was not observed in the OVA-sensitized mice with exposure to OVA and treated with tofacitinib in 7th day. (HE staining).

The concentrations of IL-4, IL-5, IL-6 and TGF-beta in BAL fluids reduced significantly in the tofacitinib treated group. The pathological scores reduced significantly in the tofacitinib treated group compared to the positive control group. Intra luminal infiltration and proliferation of Ki67 positive myofibroblasts, IL- 6 positive cell and $\alpha$-SMA positive cells in pulmonary arteries were reduced dramatically in the tofacitinib treated group compared to the positive control group.

Conclusion: Tofacitinib suppressed pulmonary vascular remodeling in a murine model of allergic vasculitis with eosinophil infiltration. Tofacitinib is a hopeful therapeutic drug for Eosinophilic granulomatosis with polyangiitis.

Disclosure of Interests: None declared

DOI: 10.1136/annrheumdis-2019-eular.4151

\section{SAT0244 EXPANDING SPECTRUM OF ADENOSINE DEAMINASE 2 (DADA2) MANIFESTATIONS: EXPERIENCE 13 PATIENTS FROM INDIA}

Aman Sharma ${ }^{1}$, Gsrsnk Naidu ${ }^{1}$, Ramesh Jois ${ }^{2,3}$, Chengappa Kavadichanda ${ }^{4}$, Rajkiran Dudam ${ }^{5}$, Sanjay Jain ${ }^{1}$, Vir Negi ${ }^{4}$, Pui.Y Lee ${ }^{6}{ }^{1}$ Post Graduate Institute of Medical Education and Research, Chandigarh, Internal Medicine, Chandigarh, India; ${ }^{2}$ Vikram Hospital, Bengaluru, India; ${ }^{2}$ Vikram Hospital, Bengaluru, India; ${ }^{4}$ Jawaharlal Institute of Postgraduate Medical Education and Research, Puducherry, India; ${ }^{5}$ Hyderabad Rheumatology Center, Hyderabad, India; ${ }^{6}$ Boston Children's hospital, Boston, United States of America

Background: Deficiency of adenosine deaminase 2 (DADA2) is a recently described hereditary autoinflammatory disease with limited published literature [1-5]. There are no previous case series published from Asia.

Objectives: To describe the clinical features and treatment responses of patients diagnosed with DADA2 from India.

Methods: Patients diagnosed with DADA2 at 4 different centres in India were included. Details of clinical features, laboratory investigations, ADA2 activity, genetic analysis of CECR 1 gene, therapy received and outcomes were noted.

Results: A total of 13 patients (12 Indians and one Syrian) were diag nosed with DADA2 between April 2017 and January 2019. The median age was 22 years (range: $7-39$ years) and 8 (61.5\%) patients were males. The diagnosis was made at a median duration of 30 months (range: 1-360 months) from the onset of first symptom. Figure 1 shows the various clinical manifestations. One patient had unknown manifestation of bad obstetric history mimicking APLA syndrome. ESR (median: 49.5 $\mathrm{mm} / \mathrm{hr}$; range: $15-130 \mathrm{~mm} / \mathrm{hr}$ ) was raised in all except one patient who was in remission at the time of diagnosis. CRP value was available in 11 patients and was elevated in all (median: $51.4 \mathrm{mg} / \mathrm{L}$; range: 17.6-140 $\mathrm{mg} / \mathrm{L})$. Immunoglobulin levels assessed in 4 patients were normal. Neuroimaging done in nine patients showed infarcts in 5 , bleed in 3 , multiple aneurysms and features of PRES in one patient each. Abdominal microaneurysms were noted in 5 patients. Arterial occlusion of peripheral limb vessels was noted in 2 patients. ADA2 activity was assessed in 9 patients and all had nearly undetectable activity. CECR1 gene mutation analysis was available in 9 patients: 8 patients were homozygous for $p$ (Gly47Arg) missense mutation at exon 2 of CECR1 gene while one patient displayed compound heterozygosity for two novel mutations, $P$ (Leu188Val) in exon 4 and C.753+2 $\mathrm{T}>\mathrm{A}$ in intron 4 of CECR1 gene. Eight patients were started on anti-TNF drugs (adalimumab in 7 and etanercept in 1). One patient required higher doses of adalimumab (40mg followed by $120 \mathrm{mg}$ at week 1 and $80 \mathrm{mg}$ at week 2) for severe gastrointestinal involvement but later died due to disseminated herpes infection. Rest of the seven patients started on anti-TNF drugs achieved disease control. Our patients had CNS, eye and Gl manifestations lower than those reported by Zhou et $\mathrm{al}^{1}$ but higher than those reported by Nanthapisal et $\mathrm{al}^{2}$ and Navon Elkan et $\mathrm{al}^{4}$

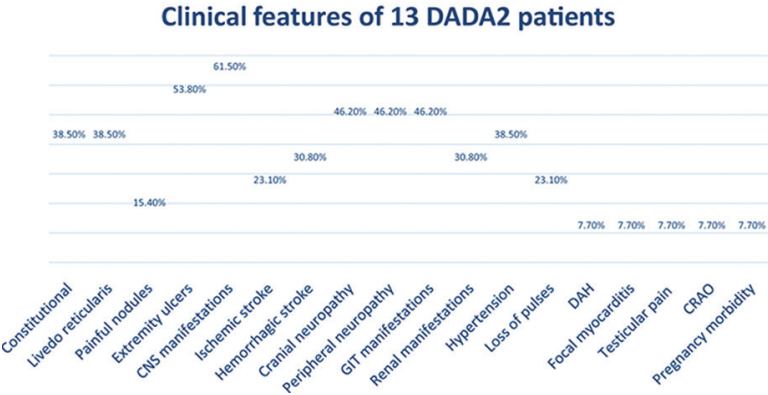

Figure 1. Clinical manifestations noted in our cohort of 13 patients with DADA2. 